\section{Towards optimal cut-off trough levels of adalimumab and etanercept for a good therapeutic response in rheumatoid arthritis. Results of the INMUNOREMAR study}

We read with great interest the paper by Chen et $a l^{1}$ analysing the relationship between therapeutic response to adalimumab and etanercept and serum drug trough levels in 70 patients with rheumatoid arthritis (RA). The authors confirmed a positive association between drug levels and European League Against Rheumatism (EULAR) response. Good responders showed significantly higher drug levels than moderate or poor responders, in line with other studies. ${ }^{2} 3$ The authors calculated (receiver operating characteristic curves) highly sensitive and specific cut-off trough levels of response $(1.274$ and $1.046 \mu \mathrm{g} / \mathrm{mL}$ for therapeutic response to adalimumab and 1.242 and $0.800 \mu \mathrm{g} / \mathrm{mL}$ for etanercept at 6 and 12 months, respectively). The study also highlights the importance of establishing an optimal cut-off for the prediction of clinical response. However, these values require confirmation in other studies and populations to be used in clinical practice.

We analysed serum trough levels of adalimumab and etanercept in 127 Caucasian patients with RA $(81.9 \%$ women, median age 61 years, median disease duration 13 years, duration of antitumour necrosis factor therapy 60 months). The cross-sectional study used the same bridging ELISA commercial kit and methodology (Promonitor, Progenika, Biopharma, Spain) as Chen et al. ${ }^{1}$ Fifty-four patients received adalimumab and 73 etanercept $(29.9 \%$ on monotherapy and $27.6 \%$ on reduced doses of biologics). Ninety-one patients came from the prospective, multicentre INMUNOREMAR (Immunogenicity, remission and arthritis) study $^{4}$ (all with disease activity score in 28 joints (DAS28) $\leq 3.2$ at baseline) and 36 were patients with RA in whom immunogenicity was assessed clinically due to active disease (all DAS28 >3.2). Seventy-one (55.9\%) patients were in clinical remission (DAS28 $\leq 2.6$ ). The accuracy and discriminatory capacity of adalimumab and etanercept trough levels were assessed by ROC curves (area under the curve (AUC)) for remission (DAS28 $\leq 2.6$ ). Serum levels of adalimumab and etanercept were significantly higher in patients in remission than in those who were not (median (P25-P75) adalimumab $6.9 \mu \mathrm{g} / \mathrm{mL}(2.7-$ 12) vs $0.5(0.1-1), \mathrm{p}<0.001$, etanercept $2.3 \mu \mathrm{g} / \mathrm{mL}(1.5-3.1)$ vs $0.8(0.4-1.8), \mathrm{p}<0.001)$. For both drugs, drug levels of patients in remission were very close to those observed by Chen $e t$ al in patients with a good EULAR response at month 6 (adalimumab $6.5 \mu \mathrm{g} / \mathrm{mL}(3-11.5)$ and etanercept $2.3 \mu \mathrm{g} / \mathrm{mL}(1.4-2.3)$ ).

In our study, analysis of accuracy with remission by DAS28 as the reference variable showed an AUC for adalimumab of 0.81 (95\% CI 0.68 to 0.94 ) and etanercept of 0.747 (95\% CI 0.68 to 0.85$)$. Trough levels with the greatest discriminative capacity for remission were $1.336 \mu \mathrm{g} / \mathrm{mL}$ for adalimumab (sensitivity $81.9 \%$, specificity $81 \%$ ) and $1.56 \mu \mathrm{g} / \mathrm{mL}$ for etanercept (sensitivity $71.1 \%$, specificity $71.4 \%$ ). These cut-offs were similar to those proposed by Chen et al, especially in the case of adalimu$\mathrm{mab}(1.274 \mu \mathrm{g} / \mathrm{mL}$ for adalimumab and $1.046 \mu \mathrm{g} / \mathrm{mL}$ for etanercept). The cut-off values observed in the two studies are lower than those observed in other studies, possibly reflecting detection methods or patient characteristics. ${ }^{2} 356$

In summary, the results of a study in Caucasian patients with RA treated with adalimumab and etanercept that used a different approach (comparing patients with and without remission by DAS28), but the same commercial ELISA bridging test, largely confirm the results obtained by Chen et al in Asian patients. This underlines the relationship between trough serum levels of the two drugs and a good therapeutic response and is further evidence in the search to find a cut-off drug level of response that could help optimise the management of patients with RA receiving these drugs in clinical practice.

Raimon Sanmarti, Jose Inciarte-Mundo, Paula Estrada-Alarcon, Maria Garcia-Manrique, Javier Narvaez, Jesus Rodriguez-Moreno, Antoni Gomez-Centeno, Mariona Pascal, Jordi Yagüe

Rheumatology Department, Hopsital Clinic de Barcelona, Barcelona, Spain

Correspondence to Dr Raimon Sanmarti, Rheumatology Department, Hopsital Clinic de Barcelona, Villarroel 170, Barcelona 08036, Spain; sanmarti@clinic.cat

Funding Study funded by an unrestricted grant from Pfizer (WS2392358).

Competing interests None.

Provenance and peer review Not commissioned; internally peer reviewed.

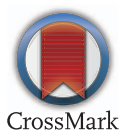

To cite Sanmarti R, Inciarte-Mundo J, Estrada-Alarcon P, et al. Ann Rheum Dis 2015;74:e42.

Received 2 March 2015

Accepted 3 March 2015

Published Online First 24 March 2015

\section{S Linked}

http://dx.doi.org/10.1136/annrheumdis-2015-207543

Ann Rheum Dis 2015;74:e42. doi:10.1136/annrheumdis-2015-207530

\section{REFERENCES}

1 Chen D-Y, Chen YM, Tsai W-C, et al. Significant associations of antidrug antibody levels with serum drug trough levels and therapeutic response of adalimumab and etanercept treatment. Ann Rheum Dis 2015;74:e16.

2 Jamnitski A, Bartelds GM, Nurmohamed MT, et al. The presence or absence of antibodies to infliximab or adalimumab determines the outcome of switching to etanercept. Ann Rheum Dis 2011;70:284-8

3 Bartelds GM, Wijbrandts CA, Nurmohamed MT, et al. Clinical response to adalimumab: relationship to anti-adalimumab antibodies and serum adalimumab concentrations in rheumatoid arthritis. Ann Rheum Dis 2007;66:921-6.

4 Sanmarti R, Inciarte J, Estrada Alarcon P, et al. Immunogenicity of anti-TNF antagonists in patients with rheumatoid arthritis or polyarticular psoriatic arthritis in clinical remission or low disease activity: The INMUNOREMAR study. Ann Rheum Dis 2014;73(Suppl 2):479.

5 Rosas J, Llinares-Tello F, de la Torre I, et al. Clinical relevance of monitoring serum levels of adalimumab in patients with rheumatoid arthritis in daily practice. Clin Exp Rheumatol 2014;32:942-8.

6 Pouw MF, Krieckaert CL, Nurmohamed MT, et al. Key findings towards optimising adalimumab treatment: the concentration-effect curve. Ann Rheum Dis. 2015;74:513-18. 